\title{
Frequency dependence of magnetothermal properties for magnetic fluid and magnetically functionalized implants
}

\author{
Salakhova R.T. ${ }^{1}$, Vylegzhanin A. G. ${ }^{1}$, Kashtanov E.A. ${ }^{1}$, Zverev V.I. ${ }^{1}$, \\ Müller R. ${ }^{2}$, F.D. de Sena Pereira ${ }^{3}$, Parfenov V.A. ${ }^{3}$, V.A. Mironov V.A. ${ }^{3}$, \\ Kritskaya E.A. ${ }^{4}$, Pyatakov A.P. ${ }^{I}$, Markov V.K. ${ }^{5}$, Malyshev A.Y ${ }^{5}{ }^{5}$, Kamilov K.I ${ }^{I}$, and Tishin A.M ${ }^{1,5}$
}

\author{
${ }^{1}$ M.V. Lomonosov Moscow State University, Leninskie gori street 1, Moscow, Russia \\ ${ }^{2}$ Institute of Photonic Technology, Albert-Einstein-Straße 9, Jena, Germany, \\ ${ }^{3} 3 \mathrm{D}$ Bioprinting Solutions Lab, 68/2, Kashirskoe highway, Moscow, Russia \\ ${ }^{4}$ LLC Shell, Novinskii boulevard 31, Moscow, Russia \\ ${ }^{5}$ AMT\&C Group, Promishlenaya street 4, Troitsk, Russia,
}

\begin{abstract}
Heating of the magnetic nanoparticles in AC magnetic field is the effect promising for application in medicine. The mechanisms of heating in AC-magnetic field implies nontrivial dependence of the power dissipated by magnetic nanoparticles on frequency. With the use of a reconfigurable experimental setup, this frequency-dependent magnetic heating was measured on two characteristic examples: the magnetite nanoparticles conventionally used in medicine and polymer coating with micrometer sized magnetite particles. The saturation of the heating power with frequency is shown that is more pronounced for the second case of microparticles.
\end{abstract}

\section{Introduction}

Magnetic nanoparticles (MNp) have attracted attention in modern medicine due to their possibility of injecting and accumulating in the target tissue. Heating of MNp in alternating (AC) magnetic field leads to the therapeutic effect either through direct thermal treatment $[1,2]$ or due to the heat-assisted release of the drug carried by nanoparticles [3]. The magnetically functionalized implants, which consist of polymeric matrices and $\mathrm{MNp}$, can also be used as effective method to prevent undesired effect of inflammation or cell proliferation.

There are three types of heating mechanisms of magnetic nanoparticles in AC-magnetic field: Brown relaxation, Néel relaxation, and hysteresis losses. Brown relaxation implies the nanoparticles have freedom of rotation in the fluid and their rotation in the external AC magnetic field is accompanied with energy dissipation due to the viscous damping. In contrast to the Brown relaxation, the Néel relaxation mechanism is based on the rotation of the magnetic moments of the nanoparticles in AC magnetic field with energy dissipated during the relaxation of the magnetic moments around the equilibrium position. The Néel relaxation prevails for immobilized magnetic nanoparticles or particles with the large time of Brown relaxation. Finally the hysteresis losses are dominant in multidomain particles and associated with the power dissipated during the cycle of magnetization reversal.

A common parameter for quantitative characterization of magnetic heating is the Specific Absorption Rate (SAR), i.e. the ratio of the power dissipated to the mass of the particles. This value is of clinical importance for calculation of particle concentration and field exposure time.

Since all of those three above mentioned mechanisms are related to some charactersitic relaxation time [4] or hystersis cycle period, SAR should be strongly dependent on the frequency of the AC magnetic field. Thus it is crucial to have the setup with easily changeable frequency range.

In this paper the special reconfigurable experimental setup was developed to study the frequency dependence of the magnetothermal properties on two characteristic examples: the magnetic fluid and magnetically functionalized polymer implants.

\section{The experimental setup and measurements procedure}

The experimental setup for calorimetric SAR measurements consists of an AC magnetic field module, a water cooling system and a PC data acquisition system (fig.1). The AC magnetic field module comprises the $400 \mathrm{~W}$ power supply, the generator connected in series with the oscillating circuit that includes the reconfigurable capacitor system and the main part of the setup: the solenoid providing magnetic field 100 Oe (its stability is controlled in real time with a test coil) in the working volume with the inner diameter $7 \mathrm{~mm}$. Due to the reconfigurable capacitor system there is a possibility to adjust the frequency in the vicinity of the oscillating circuit resonance frequencies corresponding to the set of values: $150,200,250,300 \mathrm{kHz}$. 


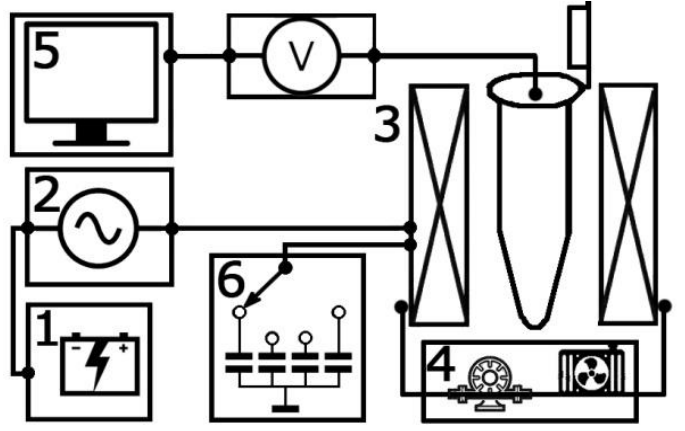

Fig. 1. Equipment for SAR measurement.1. Power supply; 2. generator; 3 . solenoid; 4. water cooling system ; 5 . data acquisition and monitoring system, 6. reconfigurable capacitor system.

During the measurements, the test tube with a sample and thermocouple was placed into homogeneous magnetic field region deep inside the coil.

SAR was calculated according to the definition:

$$
S A R=C \frac{M}{m} \frac{d T}{d t}
$$

where $C$ is water specific heat capacity, $d T / d t$ is the rate of heating in the absence of heat losses, $M / m$ is the ratio of the water mass to the mass of the sample (powder of nanoparticles or a polymer implant functionalized).

Due to the non-adiabatic measurement condition the actual slope of the heating curve differs from $d T / d t$ in eq.(1). Generally speaking, the detailed thermodynamic model of the heat exchange mechanisms in experimental setup is needed to calculate the energy losses [5]. We used the empirical approach that is the variation of the "corrected slope" method [6]: to obtain the adiabatic value of $d T / d t$ at any given temperature in the real environment one should sum the absolute values of slopes of the heating and cooling curves at this temperature.

To exclude the parasitic effects related to the heating of the coil and the inductive interference in thermocouple the control measurements with distilled water without samples were carried out.

\section{Results}

In this part we studied the dependence of heating dynamics on frequency for two samples: the magnetic fluid of magnetite nanoparticles conventionally used in medicine [7] and the polymer implant coating with magnetite microparticles embedded in it.

\subsection{Suspension of magnetite@dextran nanoparticles}

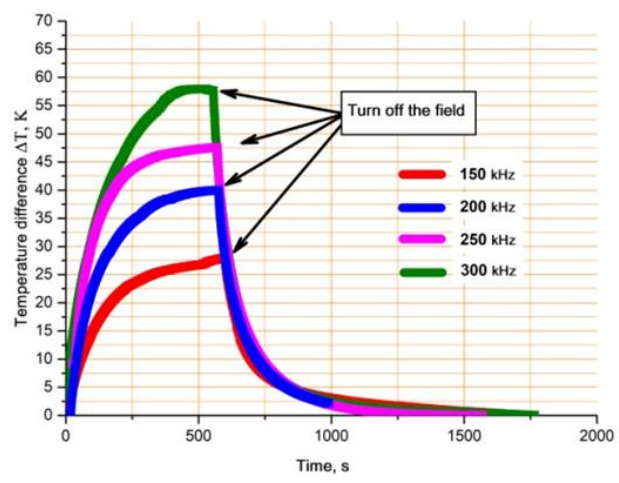

Fig. 2. The heating/cooling curves for suspension of magnetite nanoparticles at various frequencies of $\mathrm{AC}$ magnetic field.

The set of heating/cooling curves corresponding to various magnetic field frequencies for magnetite nanoparticles water suspension is shown in Fig 2. The magnetite nanoparticles with dextran coating (core diameter $15 \pm 2 \mathrm{~nm}$, and hydrodynamical diameter about $100 \mathrm{~nm}$ ) were prepared by wet chemical precipitation method followed by coating procedure with biocompatible carboxymethyldextran (for details, see [7]). The sample was prepared by stirring $20 \mathrm{mg}$ of nanoparticles in $0.1 \mathrm{ml}$ of distilled water. The amplitude of AC magnetic field was 100 Oe. One can see the growth of the ultimate temperature with the frequency of magnetic field.

\subsection{Polymer matrix with embedded magnetite microparticles}

The analogues heating/cooling curves for functionalized polymer implant is shown in fig.3. It was the electrospun polyurethane matrices embedded with magnetite particles $(3 \% \mathrm{v} / \mathrm{v})$. The particles were prepared by co-precipitation method from aqueous solution with a $2 \mathrm{Fe}(\mathrm{III}): 1 \mathrm{Fe}(\mathrm{II})$ mole ratio by hydrolyzing in $25 \%$ ammonium hydroxide solution. The average size of particles was $650 \mathrm{~nm}$ (size dispersion $350 \mathrm{~nm}$ ). The sample was merged into $0.5 \mathrm{ml}$ of water. The sample size, thickness and mass were $2 \times 2 \mathrm{~cm}, 0.25$ $\mathrm{mm}$, and $60 \mathrm{mg}$, respectively. The amplitude of $\mathrm{AC}$ magnetic field was 100 Oe. The saturation of the ultimate temperature with frequency is clearly seen. 


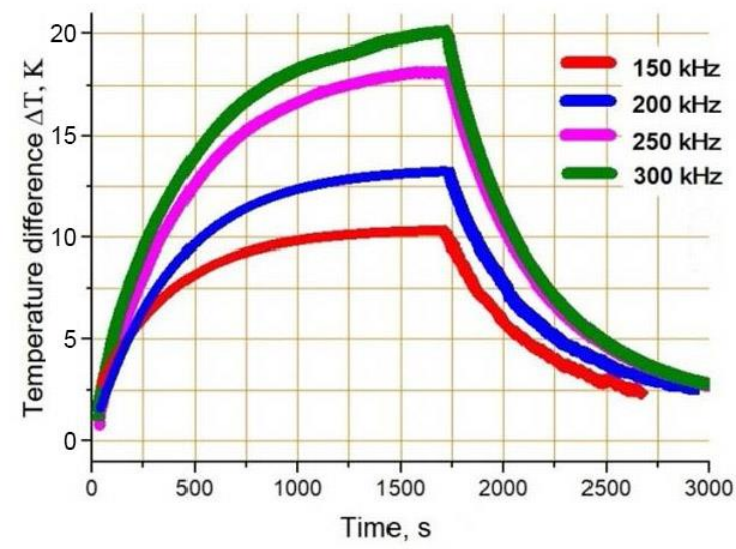

Fig. 3. The heating/cooling dynamics at various frequencies for magnetite microparticles in polymer implant coating.

\section{Discussion}

To characterize the heating of the samples quantitatively, the value of SAR according to eq. 1 was calculated. The temperature increase rate $d T / d t$ was obtained by following the procedure described in section 2 .

We also approximate the heating/cooling curves with the following equation, corresponding to the time evolution of the temperature [1]:

$$
\Delta \mathrm{T}(\mathrm{t})=\left.\tau_{R} \frac{d T}{d t}\right|_{t=0}\left(1-\exp \left(-\frac{t}{\tau_{R}}\right)\right)
$$

where $\tau_{R}$ is a relaxation constant which depends on the heat capacity, the surface of the sample, and the heat transfer coefficient between the sample and the medium. The value $d T / d t$ obtained from approximation of the experimental data with the dependence of eq. (2) gives the same results as the corrected slope method described in section 2. That means that we deal with conventional magnetic heating far from the blocking temperature, Curie point and other self-regulated mechanisms limiting the maximum temperature [2].

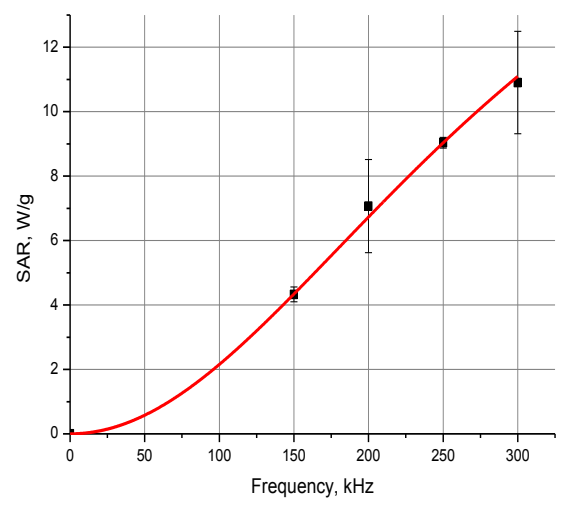

Fig. 4. The heating rate dependence on frequency of the AC field for suspension of magnetite nanoparticles.
The frequency dependences of SAR for the samples are shown in Fig.4 and 5.

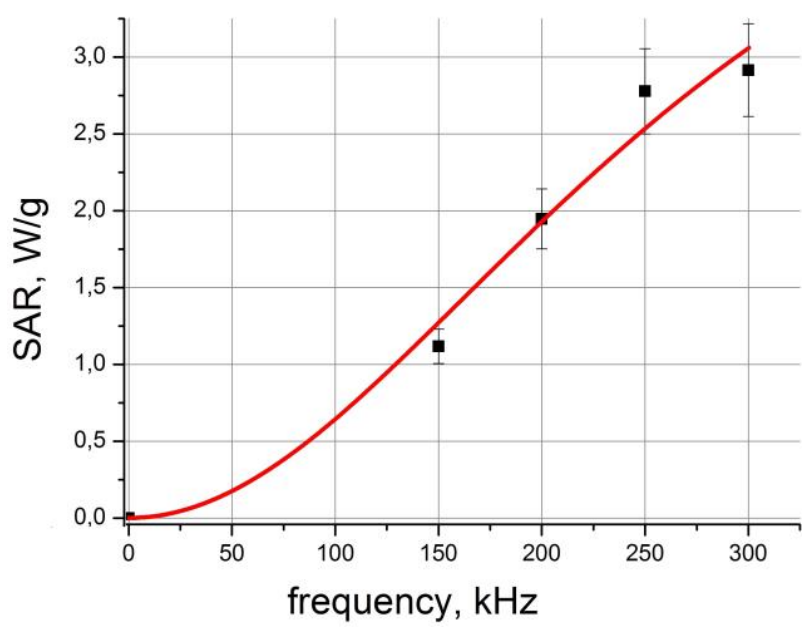

Fig. 5. The heating rate dependence on frequency for microparticles in polymer implant coating.

It should be noted that the values of SAR was calculated per unit of mass of the sample (magnetic part and the polymer coating) so they cannot be used for comparison of heating ability of bare magnetite nanoparticles. However we can compare the character of the frequency dependence (the asymptotics, the saturation etc). The SAR is increasing with the frequency for both of the samples, but the saturation of the dependence for the second sample is more prominent.

To gain insight into the origin of this saturation the frequency dependence of various heating mechanism should be analyzed. The naive approach to the problem supposes the linear dependence of SAR on frequency since a certain amount of heat is released in every cycle of AC magnetic field. However, the characteristic time of the reaction of the particles on external magnetic field should be taken into account. The more accurate approach implies the dependence saturating with the frequency [4]:

$S A R=C \cdot \frac{(2 \pi f \tau)^{2}}{1+(2 \pi f \tau)^{2}}$

where $f$ is the AC magnetic field frequency, $C$ is proportionality constant, and $\tau$ is characteristic time of the magnetization relaxation or hysteresis reversal process.

Approximation of the data in fig.4 by eq. (3) gives the value for relaxation constant $510 \pm 10$ ns. The analogous approximation of the SAR frequency dependence gives somewhat higher value $600 \pm 50 \mathrm{~ns}$ and the approximation seems to be less accurate in this case (red curve in Fig.5).

The characteristic time strongly depends on the size of the particles, it also depends on the mechanism of heating. The Brown and Neel mechanism are predominant for magnetic nanoparticle suspension, while they are 
irrelevant for magnetic microparticles embedded into polymer. Since the particles are immobilized (that eliminates Brown heating) and too large for Neel relaxation the most probable mechanism is hysteresis losses, that can be the reason why the superparamagneticlike dependence of eq.3 not fully describes the experimental data. Intriguingly, the magnetic heating rate is not just a linear function of the frequency. The number of remagnetization cycles per second is not the only factor that matters: the energy released in one hysteretic cycle is also the function of frequency.

\section{Conclusions}

Theory of magnetic heating in AC field predicts nontrivial dependence on frequency with quadratic dependence at low frequencies, linear law in the intermediate frequency range and saturation of heating at high frequencies. To explore this frequency dependence the reconfigurable experimental setup is constructed that enables to measure the magnetic heating in $100 \mathrm{Oe} \mathrm{AC}$ magnetic field at frequencies 150, 200, 250 , and $300 \mathrm{kHz}$. The frequency dependence of magnetic heating shows the sublinear dependence of magnetic heating in high frequency range. The demonstrated saturation of heating with the frequency more pronounced for larger particles implies that the "naive" notion of magnetic heating rate proportional to the frequency is not valid both for Neel and hysteretic mechanism of magnetic heating.

The support of The Foundation for Assistance to Small Innovative Enterprises in Science and Technology (project \#C1-21200) is acknowledged .

\section{References}

1. B. Thiesen, A. Jordan. Int. Journal of Hyperthermia.; 24(6), 467 (2008)

2. E. A. Périgo, G. Hemery, O. Sandre, D. Ortega, E. Garaio, F. Plazaola, and F. J. Teran, Appl. Phys. Rev. 2, 041302 (2015)

3. Ch. S.S.R. Kumar, F. Mohammad, Advanced Drug Delivery Reviews, 63, 789 (2011).

4. R. E. Rosensweig, JMMM, 252, 370 (2002).

5. M. Coisson, G. Barrera, F. Celegato et al, Biochimica et Biophysica Acta, 1861(6), 1545 (2017).

6. R.R. Wildeboer, P. Southern, Q.A. Pankhurst J. Phys. D: Appl. Phys. 47, 495003 (2014).

7. S. Dutz, W. Andra, R. Hergt et al, JMMM, 311, 51 (2007) 